Running Head: Frequency in reasoning

PSYCHOLOGICAL REPORTS (2005) vol. 97, pp. 691-695 ISSN: 0033-2941.

\title{
FREQUENCY FORMAT
}

\section{FACILITATES REASONING IN SIMPLE NUMERICAL TASKS ${ }^{1}$}

\section{RAFFAELLA MISURACA AND MAURIZIO CARDACI}

Dipartimento di Psicologia

Università degli Studi di Palermo

\footnotetext{
${ }^{1}$ Address enquiries to Raffaella Misuraca, Dipartimento di Psicologia, Università di Palermo, Viale delle Scienze, Edificio15, 90128 Palermo, Italy, or e-mail (rmisura@unipa.it).
} 
Summary. This study examined whether is it easier to reason in terms of frequencies or with percentages for simple numerical tasks. Research on probabilistic reasoning has shown that humans can draw correct inferences when problems are presented in terms of natural frequencies, but not when in percentages. Whether the same effect can be observed in other numerically simple tasks which are not probabilistic was studied with 40 undergraduate students who volunteered for the experiment (13 men, 27 women, M age of $23 \mathrm{yr}$.). In a simple numerical task involving frequencies or percentages $(N=20)$, their performance showed representation in frequencies facilitates the task.

Keywords: Representation of information; Natural frequencies; Evolutionary psychology. 
Recent literature on probabilistic reasoning has largely investigated the facilitating effect found with use of natural frequencies. In particular, Gigerenzer and Hoffrage (1995) showed that representing information in terms of natural frequencies instead of percentages enhances one's performance on probabilistic tasks (see also Cosmides \& Tooby, 1996). In their original experiments, the authors used two versionsone with probabilities and one with frequencies — of the same reasoning task. To illustrate, we present below the Disease problem adapted from Eddy (1982).

\section{Standard Probability Format}

The probability of breast cancer is $1 \%$ for women at age 40 who participate in routine screening.

If a woman has breast cancer, the probability is $80 \%$ that she will get a positive mammography.

If a woman does not have breast cancer, the probability is $9.6 \%$ that she will also get a positive mammography.

A woman in this age group had a positive mammography in a routine screening. What is the probability that she actually has breast cancer? $\%$

\section{Standard Frequency Format:}

Ten of every 1,000 women at age 40 who participate in routine screening have breast cancer.

Eight of every 10 women with breast cancer will get a positive mammography. Out of every 990 women without breast cancer 95 will also get a positive mammography.

Here is a new representative sample of women at age 40 who got a positive mammography in routine screening. How many of these women do you expect actually to have breast cancer? out of

The first standard probability format refers to single-event probabilities expressed as percentages: all information is in the form of probabilities attached to a single person, and the task is to estimate this single-event probability. To solve this task in a Bayesian 
way, people need to update prior opinions (prior probability $P(E)$ ) about the event $E$, in light of new data $D$ (posterior probability, $P(E \mid D)$ ), according to the following mathematical rule:

$$
P(E \mid D)=P(D \mid E) P(E) / P(D)
$$

On the contrary, the standard frequency format conveys frequencies in relation to a reference class of 1000 women. To solve this second version, all the information a participant needs is the number of women who had both the symptom (positive mammography) and the disease (breast cancer) and the number of women with the symptom (all the cases of positive mammography). The correct solution can be obtained by solving the following equation:

$$
P(E \mid D)=\text { Frequency }(D \& E) /[\text { Frequency }(D \& E)+\text { Frequency }(D \&-E)]
$$

or

$$
\mathrm{P}(\mathrm{E} \mid \mathrm{D})=8 /[8+95]
$$

Equation 1 and 2 are mathematically equivalent formulations of Bayes' theorem. Both produce the same result, $p(\mathrm{H} \mid \mathrm{D})=.078$. However, the frequency format has been shown to be systematically easier for participants to handle (Gigerenzer \& Hoffrage, 1995, 1999; Gigerenzer, 1996; Hoffrage \& Gigerenzer, 1998; Hoffrage, Gigerenzer, Krauss, \& Martignon, 2002; see also Cosmides \& Tooby, 1996).

Originally, Gigerenzer and Hoffrage (1995) explained this result referring to human evolutionary history. Indeed, natural frequencies, defined as the number of groupings into which a considered sample can be subdivided, represent the way in which humans have always encountered numerical information. The authors assume that in an unalphabetized world the first field of application of statistical reasoning referred to data available in a natural frequency format and not to probabilities $(p=.15)$ or percentages $(15 \%)$. 
In addition to the evolutionary explanation, the authors proposed a computational explication: the Bayesian algorithm is computationally simpler when information is represented in a frequency format rather than in a standard probability one because fewer operations must be performed. Moreover, the frequency format involves operations which can be performed on whole numbers (absolute frequencies), while the probability format involves calculations with fractions (such as percentages) (Gigerenzer \& Hoffrage, 1995).

Starting from the work of Gigerenzer and Hoffrage (1995), several experiments have been run, often producing contradictory results. For example, contrary to the conclusion of Gigerenzer and Hoffrage (1995), other authors did not find significant differences in probabilistic tasks expressed in terms of both percentages and frequencies (e.g., Macchi \& Mosconi, 1998; Johnson-Laird, Legrenzi, Girotto, Legrenzi, \& Caverni, 1999; Evans, Handley, Perham, Over, \& Thompson, 2000; Girotto \& Gonzalez, 2001, 2002). For example, Johnson-Laird, et al. (1999) showed that a presentation of data in a frequency format does not assure correct reasoning. What appears to be important is not the information format of the task but the fact that the task can be solved via the following steps: (i) inferring the probability of an event from mental models representing what is true; (ii) assuming by default that each model represents an equiprobable alternative; and (iii) making inferences about probabilities from the proportion of models in which the event occurs. Furthermore, Evans, et al. (2000) showed that tasks expressed in terms of frequencies yield better performance only if the information is presented in a manner which facilitates the construction of a mental model helpful in finding the normative solution.

In addition to this literature, there is another consideration. The facilitation in use of a frequency format is confined to probabilistic reasoning and has never been extended 
to other numerical tasks in which information could be represented as frequencies or percentages. In light of this, the aim of this research was to assess whether it is easier to perform numerical reasoning expressed in terms of frequencies rather than percentages in simple numerical tasks that are not probabilistic.

Forty undergraduate students of the University of Palermo took part as volunteers in the experiment. Thirteen were men, and 27 were women; the median age was $23 \mathrm{yr}$. None of the participants was familiar with Bayes's theorem. They were randomly assigned to two independent groups $(n s=20)$. One group received the frequency version of a simple numerical task (cf. Van de Geer, 1957; Mosconi \& D’Urso, 1974). The other group received the percentage version of the same problem. Each participant thus worked only on one task. The participants were tested individually or in small groups of two or three.

\section{Frequency version}

Pierre and Paul are two friends, each of whom has 8 ties. One day Pierre gives Paul 4 of his ties as a gift. How many more ties does Paul have than Pierre?

\section{Percentage version}

Pierre and Paul are two friends, each of whom has 8 ties. One day Pierre gives Paul 50\% of his ties as a gift. What percentage more ties does Paul have than Pierre?

To solve the above two tasks, one needs to add the gift from Pierre to Paul's initial number of ties, then subtract Pierre's remaining number of ties. The correct answer is 8 in the frequency version; $200 \%$ in the percentage version.

Chi-square analyses $\left(\chi_{1}^{2}=7.36 ; p<.01\right)$ showed a significant facilitating effect: 10 of 20 participants answered correctly in the frequency version, and only 1 out of 20 participants answered correctly in the percentage version. Thus, the frequency version elicits a substantially higher proportion of correct answers $(50 \%)$ than the percentage 
version (5\%). The relatively high contingency coefficient (.450) suggests a strong association between the two variables.

The most common error was a response of 4 in the frequency version and $100 \%$ in the percentange version. Both errors could be attributed to the focus of attention on the number of ties transferred rather than on the final quantities of ties held by Paul and Pierre (cf. Mosconi \& D’Urso, 1974). These results demonstrate that the facilitating effect of natural frequencies can be observed not only in probabilistic reasoning but also in other, simpler numerical tasks.

In conclusion, it seems that, although the frequency and the percentage versions are mathematically equivalent formulations of the same task (no matter whether probabilistic or numerical), they are not psychologically equivalent: the frequency format is systematically easier to manage. Also, these results are consistent with the conception outlined by Gigerenzer and Hoffrage (1995). 


\section{REFERENCES}

Cosmides, L., \& Tooby, J. (1996) Are humans good intuitive statisticians after all? Rethinking some conclusions from the literature on judgment under uncertainty. Cognition, 58, 1-73.

Eddy, D.M. (1982) Probabilistic reasoning in clinical medicine: problems and opportunities. In Kahneman, D., Slovic, P. \& Tversky, A. (Eds.), Judgment under uncertainty: heuristics and biases. Cambridge, UK: Cambridge Univer. Press. Pp. 249-267.

Evans, J., Handley, S. J., Perham, N., Over, D. E., \& Thompson, V. A. (2000) Frequency versus probability formats in statistical word problems. Cognition, 77, 197-213.

Gigerenzer, G. (1996) The psychology of good judgments: frequency formats and simple algorithms. Medical Decision Making, 16, 273-280.

GigerenZer, G., \& Hoffrage, U. (1995) How to improve Bayesian reasoning without instruction: frequency formats. Psychological Review, 102, 684-704.

Gigerenzer, G., \& Hoffrage, U. (1999) Helping people overcome difficulties in Bayesian reasoning: a reply to Lewis and Keren (1999) and Mellers and McGraw (1999). Psychological Review, 106, 425-430.

Girotto, V., \& Gonzalez, M. (2001) Solving probabilistic and statistical problems: a matter of information structure and question form. Cognition, 78, 247-276.

Girotto, V., \& Gonzalez, M. (2002) Chances and frequencies in probabilistic reasoning: rejoinder to Hoffrage, Gigerenzer, Krauss, and Martignon. Cognition, $84,353-359$.

Hoffrage, U, \& Gigerenzer, G. (1998) Using natural frequencies to improve diagnostic inferences. Academic Medicine, 73, 538-540. 
Hoffrage, U., Gigerenzer, G., Krauss, S., \& Martignon, L. (2002) Representation facilitates reasoning: what natural frequencies are and what they are not. Cognition, 84, 343-352.

Johnson-Laird, P.N., Legrenzi, P., Girotto, V., Legrenzi, M.S., \& Caverni, J-P. (1999) Naive probability: a mental model theory of extensional reasoning. Psychological Review, 106, 62-88.

MACCHI, L., \& Mosconi, G. (1998) Computational features vs frequentist phrasing in the base-rate fallacy. Swiss Journal of Psychology, 57, 79-85.

Mosconi, G., \& D’Urso, V. (1974) Il farsi e il disfarsi del problema. Firenze, GiuntiBarbèra.

VAn De Geer, J. P. (1957) Psychological application of information theory. Nederlands Tijdschrift voor de Psychologie en haar Grensgebieden, 12, 295-328. 